Document downloaded from:

http://hdl.handle.net/10251/81137

This paper must be cited as:

Sans-Tresserras, JÁ; Gea Pinal, J.; Gimenez Valentin, MH.; Esteve, AR.; Solbes, J.; Monsoriu Serra, JA. (2017). Determining the efficiency of optical sources using a smartphone's ambient light sensor. European Journal of Physics. 38(2):1-9. doi:10.1088/1361-6404/aa51a9.

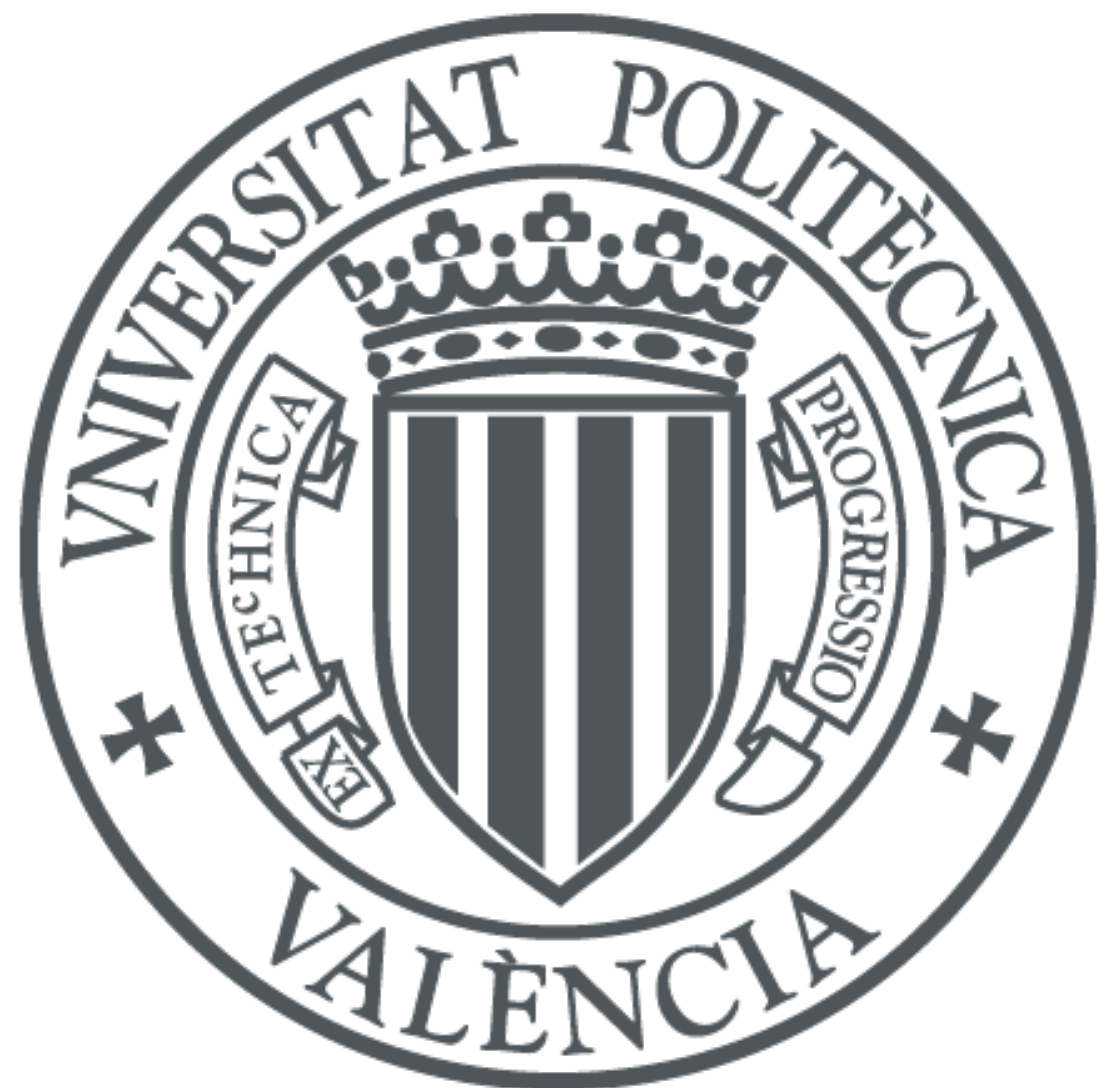

The final publication is available at

http://dx.doi.org/10.1088/1361-6404/aa51a9

Copyright European Physical Society

Additional Information 


\title{
Determining the efficiency of optical sources using the smartphone's ambient light sensor
}

\author{
J. A. Sans ${ }^{1, *}$, J. Gea-Pinal ${ }^{1}$, M. H. Gimenez ${ }^{1}$, A. R. Esteve ${ }^{2}$, J. Solbes ${ }^{2}$, J. A. \\ Monsoriu $^{1}$ \\ ${ }^{1}$ Escuela Técnica Superior de Ingeniería del Diseño, Universitat Politècnica de València, \\ Camí de Vera s/n, 46022, València, Spain \\ ${ }^{2}$ Departamento de Didáctica de las Ciencias Experimentales y Sociales, Universitat de \\ València, Avda. Tarongers 4, 46022 València, Spain
}

\begin{abstract}
This work reports the use of the smartphone's ambient light sensor as a valuable tool to study and characterize the efficiency of an optical source. Here, we have measured both luminous efficacy and efficiency of several optical sources (incandescent bulb and halogen lamp) in function of the electric power consumed and the distance to the optical detector. The illuminance of LEDs in function of the distance to the optical detector is characterized for different wavelength emissions. The analysis of the results confirm the inverse-square law of the illuminance with the detector-source distance and show a good agreement with values obtained by classical experiments. This experience will trigger the awareness of the students in terms of sustainability, light propagation and efficiency of the different optical sources.
\end{abstract}

Keywords: luminous efficiency, smartphone, ambient light sensor

\section{Introduction}

The M-learning concept is widely extended among the teaching community and recently, together with the use of familiar equipment to the students, has been explored to perform new Physics laboratory practices more attractive to them. Electronic devices such as digital cameras [1], webcams [2], optical computer mice [3,4] and game controllers [5-7] allow to determine fundamental Physics properties through the design of new and interesting experiments. Among all these electronic devices, the widespread use of smartphones by most students and the large amount of sensors contained in them offer an invaluable opportunity to perform new teaching strategies. Moreover, the constant evolution of free apps to extract the information acquired by the smartphone's sensors are supporting this initiative. This attractive tool for scientific demonstrations and experimental measurements can 'enrich educational opportunities for learners in diverse settings' [8]. Several examples on the design of new Physics laboratory practices have been recently presented showing the use of sensors contained in smartphones in physics education in different topics such as mechanics [9-13], optics [14-16], and acoustics [17,18].

Regarding the exploitation of the different optical sensors of the smartphone, M. A. Hossain and coworkers [19] proposed the use of the camera of the smartphone like a fluorimeter with a 
good agreement between their results and the values obtained by a conventional fluorimeter. L. P. Vieira et al. [20] carried out a first approach to the use of the smartphone's ambient light sensor to describe the variation of the light intensity with the inverse-square law of the distance. Using this physics law, our group [15] has characterized the variation of the light intensity to describe the simple harmonic and damped oscillatory motion with the ambient light sensor. This paper paved the path for considering the smartphone's ambient light sensor as an accessible optical detector. On the other hand, the study of the electric power consumed by several optical sources could raise students' awareness about the importance of using more efficient devices.

In the present work, we go further presenting a new laboratory experiment based on the measurement of the luminous efficiency and efficacy of several optical sources by using the smartphone's ambient light sensor in order to compare their properties. We expect that the use of their own smartphones will trigger the students' interest and motivation to perform the laboratory practice and consequently, reinforce their curiosity to carry out their own homemade experiments.

\section{Methods}

The luminous efficacy of a light source defines how well a device transforms the electrical power into luminous energy. It is determined by the equation (1), which expresses the ratio between the luminous flux $(\phi)$ and electrical power $(P)$.

$$
\eta=\frac{\phi}{P}
$$

Therefore, in the literature it is possible to find a similar parameter defined by light emitted in function of the maximum theoretical light emission $(683 \mathrm{~lm} / \mathrm{W})$ defined at $555 \mathrm{~nm}$ [21]. The choice of this wavelength is not random but is that at which the human eye is more sensitive. This value is obtained from the blackbody radiation and is called luminous efficiency, expressed as a percentage. The difference between efficacy and efficiency is not always rigorously kept in some publications, the efficacy must be always expressed on lumens/watt and efficiency is unitless.

The intensity of the light emission of the halogen and incandescent lamp changes with the electric power applied and their variation depends on the temperature reached in the filament. According to Wien's law, that is a Physical law that expresses the variation of the maximum emission wavelength with the temperature of the black-body, the maximum emission will approach to that given at $555 \mathrm{~nm}$ when the temperature of the filament increases triggering an increase in the light efficiency. The Wien's law is defined for a black-body as:

$$
\lambda_{\max }=\frac{0.0028976}{T}
$$


In the particular case of smartphones, they are usually equipped with a light sensor that allows adjusting the brightness of the display based on the environmental lighting to optimize their battery life. This light sensor uses a photodiode, in combination with a filter, to adjust its spectral sensitivity to the sensitivity of the human eye. This device is able to measure the illuminance $(E)$, which is calculated by the luminous flux $(\phi)$ per unit area $(A)$ as expressed in equation (3):

$$
E=\frac{\phi}{A}
$$

The size of the different optical sources used in this work, smaller than the detector-source distance, allows considering them as point sources. In this case, the energy in a certain region is determined by the amount of luminous flux that crosses a defined area. In the case of a point source, the luminous flux is propagated in all directions and is distributed over a spherical surface. Considering the smartphone's ambient light detector as a point detector, the illuminance measured is approximatively equal to the density of luminous flux projected by the optical source. Thus, the illuminance can be expressed as:

$$
E=\frac{\phi}{4 \pi r^{2}}
$$

When the luminous source have some preferred light emission, like in the case of light emission diodes (LEDs), the propagation is along a spherical cap, expressed as:

$$
E=\frac{\phi}{2 \pi r h}=\frac{\phi}{2 \pi r^{2} \tan \alpha} \text {. }
$$

Being $\alpha$ the apex angle determined by the manufacturer and defined by aperture angle of the lighting distribution.

The experimental measurement of these magnitudes requires a point detector or with a small surface to determine the luminous flux and consequently, the illuminance [22]. Here, we propose the simple experimental set-up shown in Figure 1 in order to determine the illuminance of different optical sources. The optical source has been placed on the optical bench in a darkened room and connected to a variable power supply in order to control the electrical power provided to it. A smartphone (Samsung Galaxy S5) has also been placed on the optical bench with the center of its light sensor facing the light bulb. We have used the 'Sensor Box for Android v5.0' free App [23] to quantitatively determine the luminous intensity that reaches the smartphone's light sensor. The illuminance provided by the optical source was measured as a function of its distance to the light sensor, and as a function of the electrical power supplied. 
In this work, we have used an incandescent bulb, a halogen lamp and four LEDs to compare their illuminance ranges and the change of their efficiency with respect to the electric power supplied.
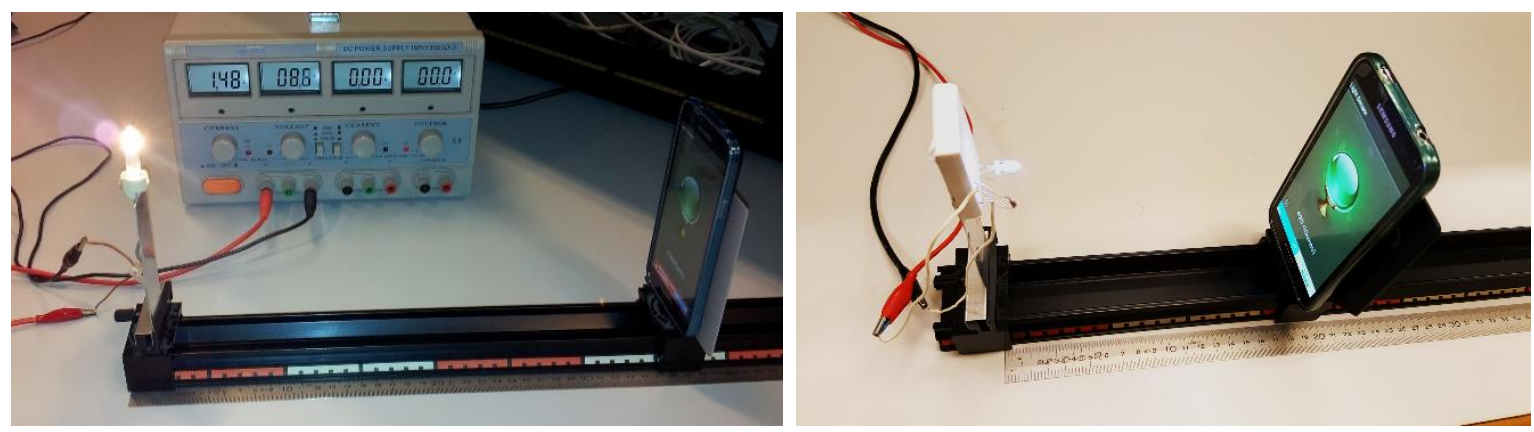

Figure 1. Experimental set-ups to measure the luminous efficiency of an optical source using a smartphone's light sensor.

\section{Results and discussions}

\section{$\underline{3.1 \text { Incandescent and halogen lamps }}$}

An incandescent bulb is formed by a wire filament through which an electric current passes, making the filament to heat up enough to radiate in the visible range. The bulb isolates the filament avoiding its oxidation by the presence of an inert environment or vacuum. Here, we have studied the illuminance of an incandescent bulb (Osram 7506) as it is the most common optical source used in a basic laboratory. The illuminance was measured as a function of the distance between the light source and the smartphone's light sensor, as well as a function of the electric power consumed. The distance range used between the light source and the smartphone's light sensor varied from $(8.4 \pm 0.1) \mathrm{cm}$ to $(30.5 \pm 0.1) \mathrm{cm}$ and the consumed electric power ranged between $(7.10 \pm 0.19) \mathrm{W}$ and $(23.1 \pm 0.3) \mathrm{W}$. The experimental illuminance data collected by the smartphone's light sensor have been used to estimate the luminous flux according to Eq. 3, considering that the luminous energy is equally distributed along spherical surfaces. The representation of the experimental illuminances at several electric powers as a function of the source-detector distance is shown in Figure 2. These experimental data display a clear quadratic dependence with distance and can be fitted using least squares. All these fits show very good correlation coefficients close to 0.99 . The large error observed in the $y$-intercept value given by the fit can be explained in terms of: i) the incandescent bulb cannot be considered as a point source at short source-detector distances and ii) the environmental light conditions, which should give a constant illuminance value. The luminous efficiency of the incandescent lamp increases with the increase of the electric current, which ranges between $1.5 \%$ and $2.3 \%$. 


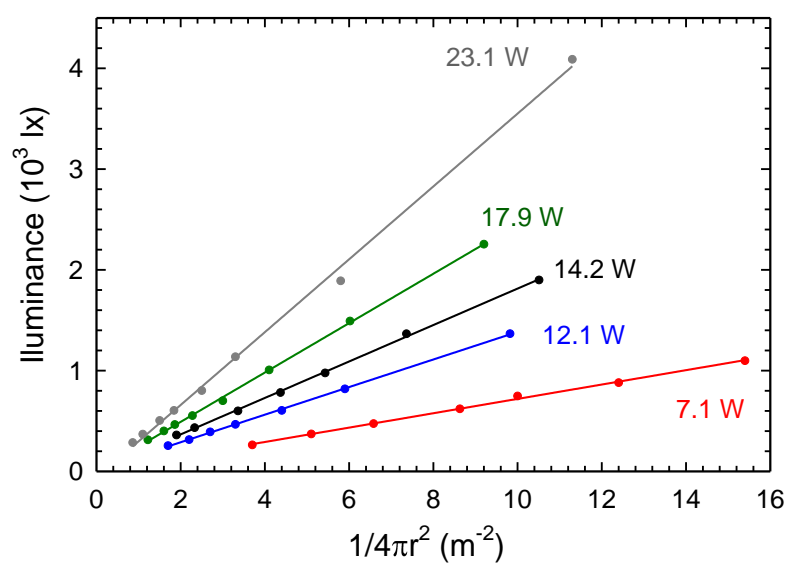

Figure 2. Experimentally measured illuminance of an incandescent bulb versus the inverse of a spherical surface of radius $r$ (symbols) for each electric power supplied. Least-square fits (lines) serve to obtain the values reported in Table I.

The difference between the halogen lamp and the incandescent bulb lies in the presence of a halogen environment inside the bulb. This produces a halogen cycle chemical reaction with the material of the filament (typically tungsten) which is evaporated and redeposited back onto the filament. Thus the lifetime of the source is extended, allowing it to operate in a high electric power range and increasing its efficacy. In this experiment, we have characterized a halogen lamp (Osram 64427S - 58663) using similar distance ranges between the light source and the smartphone's light sensor, $(8.9 \pm 0.1) \mathrm{cm}$ to $(30.0 \pm 0.1) \mathrm{cm}$, and a similar consumed electric power range, $(7.3 \pm 0.2) \mathrm{W}$ and $(21.9 \pm 0.3) \mathrm{W}$. The experimental illuminance is shown in Figure 3 as a function of the source-detector distance for several electrical powers provided. As in the case of the incandescent bulb, the halogen lamp is considered a point optical source in first approximation. The correlation factors of the least-square fits exhibit values close to 0.99 , which indicate the validity of the inverse-square law.

Equations 1 and 3 allow to determine the luminous efficacy and efficiency for each electric power provided (Table 1). The typical value of the luminous efficacy for a halogen lamp (20 $1 \mathrm{~m} / \mathrm{W}$ ) [24-26] is close to that obtained for the highest electrical power provided, which indicates the validity of the method used. The halogen lamp increases the luminous efficiency, which ranges between $1.1 \%$ and $3.4 \%$, in good concordance with the efficiency reported for tungsten halogen lamps at highest electric power in [27] (3\%). The efficiency obtained in the halogen lamp is higher than that of the incandescent bulb for similar electric power supplied, which shows that the presence of the halogen gas allows reaching higher temperatures to the filament. 


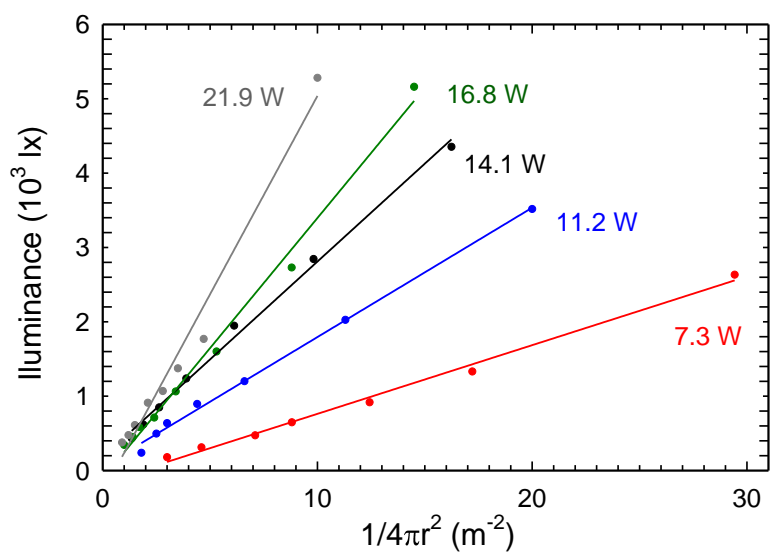

Figure 3. Experimentally measured illuminance of a halogen lamp versus the inverse of a spherical surface of radius $r$ (symbols) for each electric power supplied. Least-square fits (lines) serve to obtain the values reported in Table I.

Table I. Luminous flux, luminous efficacy, and luminous efficiency of incandescent and halogen lamps obtained for different electric powers.

\begin{tabular}{lcccc}
\hline & $\begin{array}{c}\text { Consumed } \\
\text { electric power }\end{array}$ & $\begin{array}{c}\text { Emitted } \\
\text { luminous flux } \\
(\mathbf{l m})\end{array}$ & $\begin{array}{c}\text { Luminous } \\
\text { efficacy }(\mathbf{l m} / \mathbf{W})\end{array}$ & $\begin{array}{c}\text { Luminous } \\
\text { efficiency }(\%)\end{array}$ \\
\hline & $(7.10 \pm 0.18) \mathrm{W}$ & $71.3 \pm 1.4$ & $10.0 \pm 0.5$ & 1.5 \\
& $(11.9 \pm 0.2) \mathrm{W}$ & $136.9 \pm 1.2$ & $11.5 \pm 0.2$ & 1.7 \\
Incandescent & $(14.2 \pm 0.3) \mathrm{W}$ & $181 \pm 2$ & $12.7 \pm 0.3$ & 1.9 \\
& $(17.9 \pm 0.3) \mathrm{W}$ & $245 \pm 2$ & $13.7 \pm 0.3$ & 2.0 \\
& $(23.1 \pm 0.3) \mathrm{W}$ & $361 \pm 8$ & $15.6 \pm 0.5$ & 2.3 \\
\hline \multirow{3}{*}{ Halogen } & $(7.3 \pm 0.2) \mathrm{W}$ & $92 \pm 3$ & $12.6 \pm 0.8$ & 1.9 \\
& $(11.2 \pm 0.2) \mathrm{W}$ & $174 \pm 5$ & $15.8 \pm 0.7$ & 2.3 \\
& $(14.1 \pm 0.3) \mathrm{W}$ & $263 \pm 8$ & $18.6 \pm 1.0$ & 2.7 \\
& $(16.8 \pm 0.3) \mathrm{W}$ & $350 \pm 12$ & $20.8 \pm 1.1$ & 3.1 \\
& $(21.9 \pm 0.3) \mathrm{W}$ & $530 \pm 30$ & $24.3 \pm 1.8$ & 3.6 \\
\hline
\end{tabular}

Figure 4 shows a steady increase of the luminous efficiency as a function of the electric power for the incandescent and halogen lamps. The emission in incandescent bulbs behaves like an imperfect blackbody and requires certain temperature to emit in the visible range according to the Wien's displacement law. Both of them depends on the temperature reached in the tungsten filament. The increase of the electric power supplied leads to an increase of the temperature in the filament and, consequently, to the shift of the wavelength of the maximum optical emission towards values closer to that of the maximum theoretical light emission $(555 \mathrm{~nm})$, causing an 
improvement of the efficiency [28]. The presence of a halogen environment allows to improve the efficiency of the halogen lamp with respect to the incandescent bulb due to the reconstructive effect of the halogen gas, which causes a higher filament temperature (higher illuminance) than the one obtained in the incandescent bulb. In this work, we have not exceeded the maximum electric power recommended by the manufacturer. Above the electric power recommended, the maximum optical emission could be at a higher wavelength than that at which the human eye is more sensitive $(555 \mathrm{~nm})$ or even the filament could evaporate too much degrading it. All these factors will trigger a decrease of the luminous efficiency.

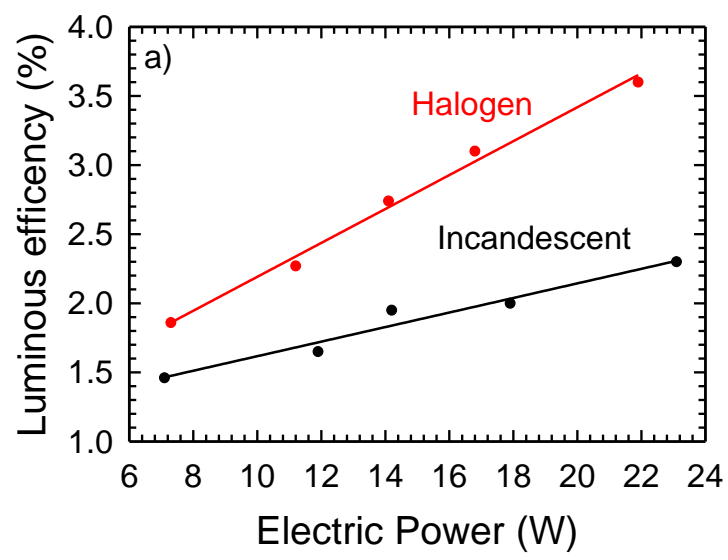

Figure 4. Measured luminous efficiency of incandescent and halogen lamps.

Besides the steady increase of the luminous efficiency with the electric power, we have also observed a linear tendency between both parameters. Above $600 \mathrm{~K}$, the temperature dependence of the tungsten filament with the electric power [29-31] follows a quasi-linear trend. This effect is due to the direct relationship between the electrical resistance of the cathode and the temperature raised to the power of $1.2[29,30]$. According to the Wien's displacement law, an almost linear behavior of the temperature with the electric power supplied leads to an almost linear behavior of the maximum emission wavelength with the same electric power. If the maximum emission wavelength of the tungsten lamp is getting closer to the wavelength where the human eye is more sensitive (555 nm), then the optical efficiency should increase in the same way. This explanation allowed us to approximate the fit of our experimental results to a linear equation.

\subsection{LEDs}

A LED consists on the junction of two doped semiconducting materials (one p-type and the other n-type) forming a diode. Applying an electric current, the electrons flows from the n- 
type semiconductor towards the p-type semiconductor but not in the reverse. The electron is recombined when meets a hole, releasing energy in the form of a photon. The optimization of this process has permitted the manufacture of ultra-bright LEDs. These optical sources offer a higher illuminance for the same electric power. LEDs cannot be considered as a point source since they show directionality in their emission. This characteristic comes defined by the aperture angle $\left(20^{\circ}\right.$ in this case for all the LEDs used) and the luminous flux is distributed over a spherical cap instead of a sphere. In this work, we have explored the efficacy of LEDs (825MR2C, 825MY8C, 825PG2C) emitting at different wavelengths and we have compared the results with an ultrabright $8 \mathrm{~mm}$ white LED [32]. The efficacy of LEDs emitting in the red, yellow and green has been obtained from the variation of the illuminance as a function of the LED-smartphone distance, and these values have been compared with those obtained for an ultra-bright LED. The characteristics of these devices are shown in Table II.

Table II. Voltage supplied to the circuit and voltage, current and electric power used by each LED together with luminous flux calculated for each LED.

\begin{tabular}{cccccc}
\hline & $\begin{array}{c}\text { Voltage } \\
\text { supplied (V) }\end{array}$ & $\begin{array}{c}\text { Voltage in } \\
\text { LED (V) }\end{array}$ & $\begin{array}{c}\text { Current } \\
(\mathbf{m A})\end{array}$ & $\begin{array}{c}\text { Electric } \\
\text { power }(\mathbf{m W})\end{array}$ & $\begin{array}{c}\text { Emitted } \\
\text { luminous } \\
\text { flux (lm) }\end{array}$ \\
\hline Green & $24.0 \pm 0.2$ & $2.4 \pm 0.1$ & $18.1 \pm 0.4$ & $434 \pm 4$ & $0.12 \pm 0.02$ \\
\hline Yellow & $24.0 \pm 0.2$ & $2.1 \pm 0.1$ & $18.3 \pm 1.0$ & $439 \pm 4$ & $0.24 \pm 0.03$ \\
\hline Red & $24.0 \pm 0.2$ & $2.0 \pm 0.1$ & $18.4 \pm 0.8$ & $442 \pm 4$ & $0.57 \pm 0.06$ \\
\hline Ultra-bright & $24.0 \pm 0.2$ & $3.4 \pm 0.1$ & $17.2 \pm 1.2$ & $413 \pm 4$ & $11.9 \pm 0.7$ \\
\hline
\end{tabular}

LED-smartphone distances are in the same range for all the LEDs studied. In particular, the distance between the light source and the smartphone's light sensor is in a range from (3.6 \pm $0.1) \mathrm{cm}$ to $(17.4 \pm 0.1) \mathrm{cm}$, similar to that of the incandescent or halogen lamp. On the other hand, the electric current consumed by the LEDs is much lower than the values supplied (see Table II). In order to avoid effects of efficiency drop [33], we have fixed the electric current to the optimal value provided by the manufacturer.

The comparison of these results with those given for incandescent and halogen lamps is inadequate since the origin of the light emission is completely different, even considering their different luminous distribution. The calculus of the efficiency obtained in LEDs from the blackbody radiation maximum is unfounded since the luminous efficacy does not depend on the temperature of the component materials (as it happens with the incandescent and halogen lamps) but the recombination processes carried out between n-type and p-type semiconductors. Thus, the values of the efficiency would be unreal. At the same time, LEDs show similar dependence with 
the distance that the incandescent and halogen lamps despite of the different light distribution. The low electric power consumed by LEDs give an average luminous efficacy higher than the incandescent and halogen lamps. Comparing LEDs emitting at several wavelengths (Figure 5), one can clearly see that the ambient light detector is optimized for low photon energies. On the other hand, the illuminance measured for the ultra-bright LED is one order of magnitude higher than the values obtained for usual LEDs, which reveals the increase of the efficiency achieved with the same electric current.

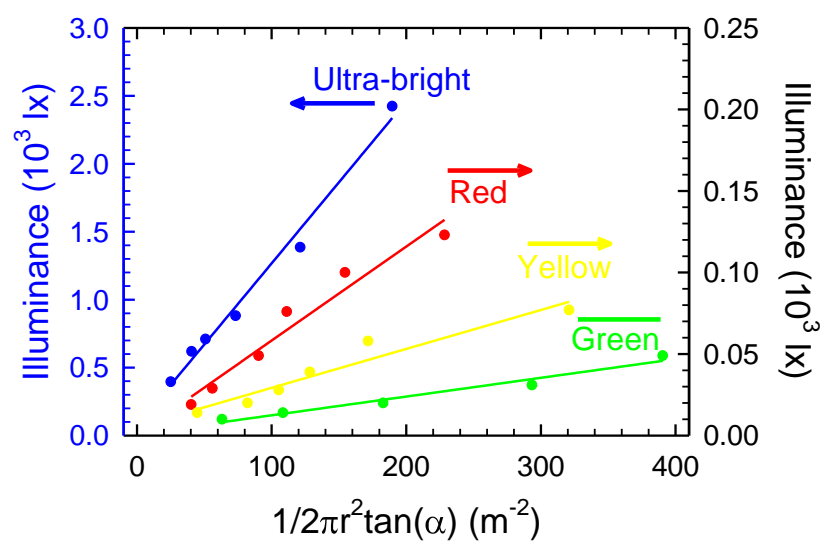

Figure 5. Illuminance as a function of the detector-source distance for LEDs emitting in different wavelengths and compared with an ultra-bright LED device.

\section{Conclusions}

We have successfully used the smartphone's light sensor and the Android application 'Sensor Box for Android' to measure the illuminance of three different optical sources. We have proved the inverse-square law dependence of the illuminance with the detector-source distance for quasi-point optical sources with isotropic light distribution and with preferred orietantion. These measurements allowed us to determine the luminous efficacy and efficiency of both incandescent and halogen lamps as a function of the electric power supplied using a least-square fit. We observed a steady increase of the luminous efficiencies obtained with the supplied electric power for both sources which has been explained in terms of blackbody emission, Wien's displacement law and the relationship between resistance and temperature given in a tungsten filament. On the other hand, we have also proved the validity of the inverse-square law dependence of the illuminance with the detector-source distance for LEDs emitting at several wavelengths. The comparison of usual LEDs with an ultra-bright LED showed an increase of two order of magnitude of the illuminance obtained by the latter. These results should stimulate students to use their smartphones to perform their own experiments at home, which will raise their awareness of the importance of the luminous efficiency. 


\section{Acknowledgements}

The authors would like to thank the Institute of Education Sciences, Universitat Politècnica de València (Spain), for the support of the teaching innovation groups MOMA and e-MACAFI, and the financial support of Project PIME-2015-B18. The authors also acknowledge the financial support of project EDU2015-69701-P by the Spanish Ministry of Economy and Competitiveness and the European Regional Development Fund. J.A. Sans acknowledges Ramon y Cajal fellowship program for financial support.

\section{References}

[1] Monsoriu J A, Gimenez M H, Riera J and Vidaurre A 2005 Eur. J. Phys. 261149

[2] Shamim S, Zia W and Anwar M S 2010 Am. J. Phys. 78433

[3] Romulo O O and Franklin K N 1997 Am. J. Phys. 651115

[4] Ng T W and Ang K T 2005 Am. J. Phys. 73793

[5] Tomarken S L et al 2012 Am. J. Phys. 80351

[6] Ballester J and Pheatt Ch 2013 Am. J. Phys. 8171

[7] Vannoni M and Straulino S 2007 Eur. J. Phys. 28781

[8] West M and Vosloo S 2013 UNESCO policy guidelines for mobile learning UNESCO Publications)

[9] Castro-Palacio J C, Velázquez-Abad L, Giménez F and Monsoriu J A 2013 Eur. J. Phys. 34, 737

[10] Castro-Palacio J C, Velázquez-Abad L, Giménez M H and Monsoriu J A 2013 Am. J. Phys. 81, 472

[11] Klein P, Hirth M, Gröber S, Kuhn J and Müller A 2014 Phys. Educ. 49, 412

[12] Vogt P and Kuhn J 2014 Phys. Teacher 52, 118

[13] Briggl J 2013 Phys. Educ. 48, 285

[14] Van Domelen D 2007 Phys. Teacher 45, 469 
[15] Sans J A, Manjón, F J, Pereira A L J, Gómez-Tejedor J A and Monsoriu J A 2013 Eur. J. Phys. 34, 1349

[16] Barreiro J J, Pons A, Barreiro J C, Castro-Palacio J C and Monsoriu J A 2014 Am. J. Phys. 82, 257

[17] Gómez-Tejedor J A, Castro-Palacio J C and Monsoriu J A 2014 Eur. J. Phys. 35, 025006

[18] Kuhn J and Vogt P 2013 Phys. Teacher 51, 118

[19] Hossain M A, Canning J, Ast S, Rutledge P J, and Jamalipour A 2015 Photonic Sensors 5, 289

[20] Vieira L P, Lara V O M, Amaral D F 2014 Rev. Bras. De Ens. de Fis. 36, 3505

[21] MacAdam D L 1950 J. Opt. Soc. Am. 40, 120

[22] Ryer A 1997 The Light Measurement Handbook, International Light Technologies, Peabody, USA

[23] https://play.google.com/store/apps

[24] Rea M S 2000 The IESNA lighting handbook: reference \& application, Illuminating Engineering Society of North America, New York, NY

[25] "Training Guide: The Basics of Efficient Lighting”. National Framework for Energy Efficiency

[26] Pumar Pérez M 2010 PhD thesis

[27] Gupta S K “Krishna's Engineering Physics: Volume III (optics)" Ed. Krishna Prakashan Media, India

[28] Hertel I V, and Schulz C-P 2014 “Atoms, Molecules and Optical Physics 1: Atoms and Spectroscopy". Ed. Springer

[29] Davis G L 1962 Nature 196, 565

[30] Forsythe W E and Worthing A G 1925 Astrophys. J. 61, 126

[31] Hill M P, Lecchini S M A, and Pethica A 1966 Trans. Faraday Soc. 62, 229

[32] 0.5W 8mm ultra-bright LED emitting 35000mcd with an apex angle of 30 degrees

[33] Iveland J, Martinelli L, Peretti J, Speck J S, and Weisbuch C. 2013 Phys. Rev. Lett. 110, 177406 